\title{
Subclones dominate at MDS progression following allogeneic hematopoietic cell transplant
}

\author{
Meagan A. Jacoby, ${ }^{1}$ Eric J. Duncavage, ${ }^{2}$ Gue Su Chang, ${ }^{3}$ Christopher A. Miller, ${ }^{1,3}$ Jin Shao, ${ }^{1}$ \\ Kevin Elliott, ${ }^{1}$ Joshua Robinson, ${ }^{1}$ Robert S. Fulton, ${ }^{3}$ Catrina C. Fronick, ${ }^{3}$ Michelle O'Laughlin, ${ }^{3}$ \\ Sharon E. Heath, ${ }^{1}$ Iskra Pusic, ${ }^{1}$ John S. Welch, ${ }^{1}$ Daniel C. Link, ${ }^{1}$ John F. DiPersio,' Peter Westervelt, ${ }^{1}$ \\ Timothy J. Ley, ${ }^{1}$ Timothy A. Graubert, ${ }^{4}$ and Matthew J. Walter ${ }^{1}$ \\ 'Department of Medicine, Division of Oncology, ${ }^{2}$ Department of Pathology \& Immunology, ${ }^{3}$ McDonnell Genome Institute, \\ Washington University School of Medicine, St. Louis, Missouri, USA. ${ }^{4}$ Massachusetts General Hospital Cancer Center, \\ Boston, Massachusetts, USA.
}

\begin{abstract}
Allogeneic hematopoietic cell transplantation (alloHCT) is a potentially curative treatment for myelodysplastic syndromes (MDS), but patients who relapse after transplant have poor outcomes. In order to understand the contribution of tumor clonal evolution to disease progression,we applied exome and error-corrected targeted sequencing coupled with copy number analysis to comprehensively define changes in the clonal architecture of MDS in response to therapy using $\mathbf{5 1}$ serially acquired tumor samples from 9 patients who progressed after an alloHCT. We show that small subclones before alloHCT can drive progression after alloHCT. Notably, at least one subclone expanded or emerged at progression in all patients. Newly acquired structural variants (SVs) were present in an emergent/expanding subclone in 8 of 9 patients at progression, implicating the acquisition of SVs as important late subclonal progression events. In addition, pretransplant therapy with azacitidine likely influenced the mutation spectrum and evolution of emergent subclones after alloHCT. Although subclone evolution is common, founding clone mutations are always present at progression and could be detected in the bone marrow as early as $\mathbf{3 0}$ and/or 100 days after alloHCT in 6 of $\mathbf{8}(\mathbf{7 5} \%)$ patients, often prior to clinical progression. In conclusion, MDS progression after alloHCT is characterized by subclonal expansion and evolution, which can be influenced by pretransplant therapy.
\end{abstract}

Authorship note: MAJ, EJD, and CSC contributed equally to this work.

Conflict of interest: The authors have declared that no conflict of interest exits.

Submitted: December 4, 2017

Accepted: February 7, 2018

Published: March 8, 2018

\section{Reference information:}

JCI Insight. 2018;3(5):e98962.

https://doi.org/10.1172/jci.

insight.98962.

\section{Introduction}

Large-scale sequencing efforts have defined the genomic landscape in multiple tumor types and have revealed that most tumors consist of multiple genetic clones (e.g., founding clone and subclones) that have differential sensitivities to therapy. Recent evidence suggests that relapsed or progressive disease in myeloid malignancies (e.g., acute myeloid leukemia [AML] and myelodysplastic syndromes [MDS]) is characterized by the emergence of one or more subclones, presumably influenced by selection pressure induced by therapy (refs. 1-4, reviewed in ref. 5). The contribution of subclones to disease progression and relapse following an allogeneic hematopoietic cell transplant (alloHCT) for MDS has not yet been comprehensively studied and will be addressed here

MDS are a heterogenous group of diseases characterized by peripheral blood cytopenias and an increased risk of progression to secondary AML, defined by a rise in the bone marrow myeloblast count to $20 \%$ or higher. MDS is a genetically heterogeneous disease (6-8) consisting of a founding clone and multiple subclones $(1,6,9)$. We and others have shown that a rare subclone can escape chemotherapy to expand at relapse or disease progression, in some cases, acquiring new mutations induced by therapy $(1,10)$.

Currently, the only potential cure for MDS patients is an alloHCT. However, posttransplant mortality remains high due to treatment-related complications and disease progression, with a 5-year estimate of overall survival of only approximately $40 \%(11,12)$. Outcomes for patients with AML or MDS with disease progression after transplant are dismal, with a 2-year overall survival for patients who relapse 
Table 1. Baseline characteristics of 9 sequenced patients

\begin{tabular}{|c|c|}
\hline Age (yr), median (range) & $61(38-66)$ \\
\hline \multicolumn{2}{|l|}{ Gender } \\
\hline Male, $n(\%)$ & $5(56)$ \\
\hline Female, $n(\%)$ & $4(44)$ \\
\hline \multicolumn{2}{|l|}{ IPSS-R } \\
\hline Very low, $n(\%)$ & $0(0)$ \\
\hline Low, $n(\%)$ & $1(11)$ \\
\hline Intermediate, $n$ (\%) & $0(0)$ \\
\hline High, $n(\%)$ & $4(45)$ \\
\hline Very high, $n(\%)$ & $3(33)$ \\
\hline Not available, $n(\%)$ & $1(11)$ \\
\hline \multicolumn{2}{|l|}{ Bone marrow blast at first banking } \\
\hline Median (range) & $7.5 \%(1 \%-35 \%)^{A}$ \\
\hline \multicolumn{2}{|l|}{ Transplant conditioning } \\
\hline Myeloablative, $n(\%)$ & $3(33)$ \\
\hline Reduced intensity, $n$ (\%) & $6(67)$ \\
\hline \multicolumn{2}{|l|}{ Progression-free survival } \\
\hline Days, median (range) & $179(93-769)$ \\
\hline \multicolumn{2}{|l|}{ WHO classification } \\
\hline MDS with excess blasts-2, $n(\%)$ & $2(22)$ \\
\hline Therapy-related MDS, $n$ (\%) & $6(67)$ \\
\hline AML with myelodysplastic changes, $n(\%)$ & $1(11)$ \\
\hline
\end{tabular}

\section{Results}

Patient characteristics and sequencing. We identified 9 patients with a history of MDS who experienced disease relapse or progression after receiving either a myeloablative $(n=3)$ or reduced-intensity $(n=6)$ alloHCT (median time to clinical progression 179 days, range 93-769 days). In 6 cases, we had additional samples immediately preceding transplant (median 18 days, range 14-36 days) available to assess tumor burden and clonal architecture at the time of transplant. Patient characteristics are as shown (Table 1 and Supplemental Table 1; supplemental material available online with this article; https://doi. org/10.1172/jci.insight.98962DS1). We performed enhanced exome sequencing (EES, exome sequencing plus deep coverage of a panel of 285 genes known to be recurrently mutated in MDS and AML) as previously described (10) to define the clonal architecture of 23 tumor (bone marrow) samples at the following clinical time points: diagnosis, immediately preceding transplant (when available), and in a progression sample after alloHCT. Skin collected at initial banking was used as a source of "normal" DNA. Sequencing the pretransplant and progression specimens, in addition to day $0 /$ first sampling specimens, allowed us to discover mutations that arose during the course of treatment. Somatic mutations were validated in the 23 discovery tumor samples (i.e., the pretransplant and progression specimens) and then genotyped in 28 additional serially banked tumor samples at various time points after alloHCT, including days 30 and 100 after alloHCT (standard clinical milestones), using a standard sequencing approach and capture probes targeting all putative single nucleotide variants (SNVs) and short insertions and deletions (indels) identified by EES (Supplemental Tables 2 and 3 and Supplemental Figure 1).

We identified 533 somatic mutations affecting the protein-coding or splice-site DNA sequence of 514 genes, including 35 recurrently mutated genes, at the first sampling time point (day 0) from 9 patients (Supplemental Table 3). One patient (unique patient number [UPN] 147457) had a substantially elevated mutational burden, with 624 mutations. An average of 17 coding and splice-site mutations per patient were present at first sampling, including an average of 3 somatic mutations in recurrently mutated genes per patient (excluding the elevated number from UPN 147457). In addition, we identified what we believe to be novel nonsynonymous somatic mutations in the centrosomal protein $192 \mathrm{kDa}$ (CEP192) gene involved in mitotic centrosome and spindle assembly in 2 of 9 patients (23) (Supplemental Table 3). 
Subclones expand or emerge, and founding clone mutations persist at disease progression after alloHCT. To determine whether subclones contribute to disease progression, we defined the clonal architecture at diagnosis and progression after alloHCT in 9 patients and immediately before transplant in 6 of these patients. We defined mutation clusters and imputed tumor clonality, as previously reported (ref. 1, reviewed in ref. 5). We defined the founding clone as the clone harboring the cluster of mutations with the highest variant allele fraction (VAF) at first sampling; as such, founding clone mutations are shared by all tumor cells. Subclones were defined as entities containing both founding clone mutations and distinct clusters of additional mutations found in only a fraction of cells (ref. 1, reviewed in ref. 5). We next examined the contribution of copy number alterations (CNAs) and loss of heterozygosity (LOH) (collectively, structural variants [SVs]) to disease progression, since these genetic events are known to be important contributors to the pathogenesis of MDS $(24,25)$ (Supplemental Table 4 and Supplemental Methods).

After defining subclonal populations using mutation VAFs, CNAs/LOH, and cytogenetic data, we compared their abundance and mutational composition prior to alloHCT and at progression after alloHCT (Figures 1 and 2 and Supplemental Figures 2-5). We identified at least 1 subclone in 7 of 9 patients prior to alloHCT (UPNs 145094, 147457, 280837, 368402, 499258, 624702, 829970) (Figure 1, Supplemental Figures 2-5, and Supplemental Tables 3 and 4). In 6 of these 7 cases, a subclone that was detectable prior to alloHCT expanded, sometimes acquiring additional genetic changes, to become the most abundant clone at disease progression after alloHCT (UPNs 147457, 280837, 368402, 499258, 624702, 829970). In the seventh case, UPN 145094, the subclone identified prior to alloHCT contracted with therapy, and a new subclone emerged after alloHCT. In all cases, founding clone mutations persisted at progression (Figure 1, A-G, Supplemental Figures 3 and 4, and Supplemental Table 3).

As an example, UPN 829970 was found to contain 4 distinct mutation clusters using SNV and indel VAFs (Figure 1, A and C). The mean VAF for cluster 1 mutations, representing the founding clone, was $45 \%$ at first sampling (i.e., $90 \%$ of bone marrow cells) and contained several genes recurrently mutated in myeloid malignancies (Figure 1, A and C). Subclonal evolution occurred when a cell derived from the founding clone acquired cluster 2 mutations, including the recurrently mutated gene KIAA1683, and formed a new subclone when a single cell containing cluster 1 and 2 mutations gained cluster 3 mutations. This third subclone was not found by standard sequencing at first sampling but was detectable at a second sampling prior to transplant at low levels by standard sequencing (day 44 mean VAF for cluster 3 mutations by standard sequencing was $2.6 \%$, i.e., present in $\sim 5 \%$ cells). Although this was a rare subclone pretransplant, it escaped eradication and emerged to become the most abundant clone at disease progression (Figure 1, A-D). In addition, a branched evolution event occurred prior to first sampling, giving rise to a subclone (containing cluster 4 mutations) that contracted at disease progression (Figure 1, A-D), suggesting that this subclone was sensitive to therapy. Although there were no mutations in genes known to be recurrently mutated in myeloid malignancies in cluster 3 , there were mutations in several protein coding genes that may have contributed to progression (Supplemental Table 3). In addition, at progression, this subclone gained additional complex SVs, including del(9q), -12, and further losses on chromosome 17 (see below and Figure 2D), all of which are known to be associated with MDS $(24,25)$. The sensitivity of our assays did not allow us to detect whether these SVs existed in cluster 3 prior to alloHCT. All founding clone mutations (i.e., cluster 1 SNVs and indels) were detectable at progression (Figure 1, A and B).

$S V$ s contribute to clonal evolution and subclone expansion after alloHCT. We found that SVs discriminated a subclone from the founding clone in 8 of 9 patients (i.e., we identified the presence of additional SVs that were not present in the founding clone) and that these SVs were typically present in the expanding/emergent subclone at progression after alloHCT (Figures 1 and 2 and Supplemental Figures 2 and 4). In 4 cases, a somatic CNA or uniparental disomy (UPD) was the only somatic mutation that defined the expanding/emergent subclone at progression (i.e., no new SNVs or indels were identified) (UPNs 145094, 435866, 499258, 574214). In 2 of these 4 cases, (UPNs 499258 and 574214) the SVs were present in the most abundant clone at disease progression (Figure 2, A-C, and Supplemental Figures 2 and 4). Overall, in the 8 patients with subclonal SVs in an expanding/emerging subclone at progression, there were only 2 cases where these SVs were unequivocally detected prior to alloHCT (UPN 280837, 499258) using sequencing and cytogenetic data (Supplemental Figures 2 and 4 and Supplemental Tables 1 and 4). These data highlight the importance of SVs in the clonal evolution of MDS at progression after alloHCT and implicate the acquisition of new SVs as late, subclonal progression events. Of note, we did not identify any definitive evidence of deletions or UPD involving the $H L A, P D-L 1$, or $P D-L 2$ genes at progression after alloHCT (Supplemental Figure 6). 
A

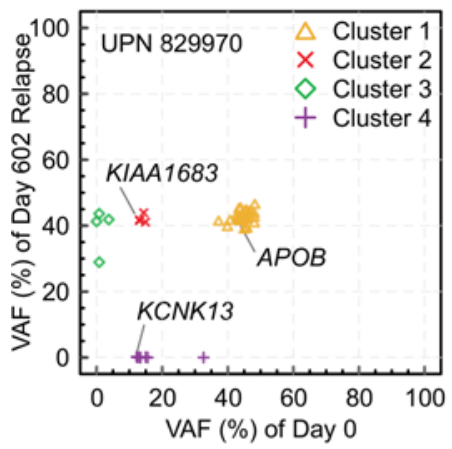

B

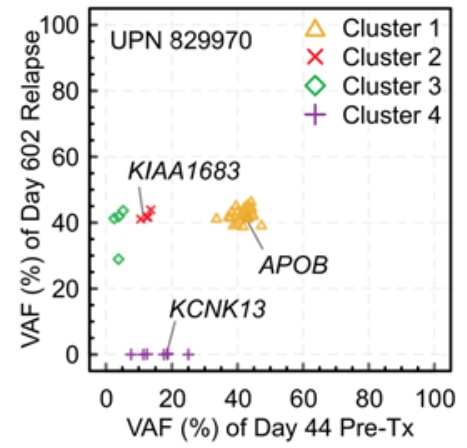

C

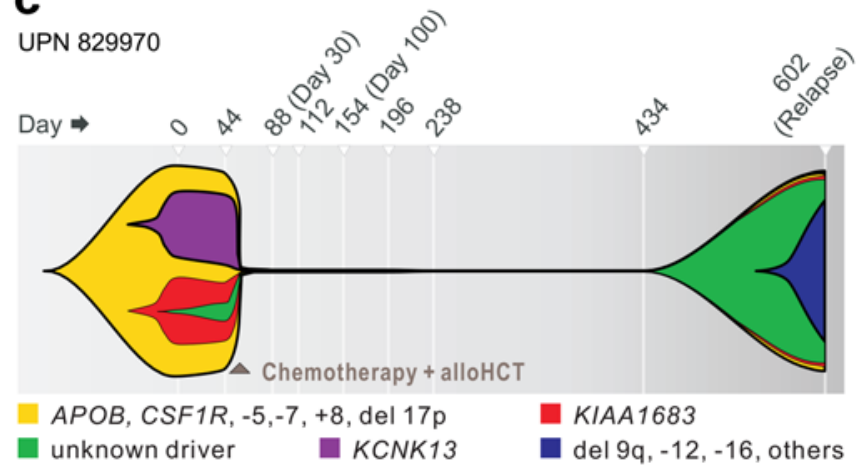

D

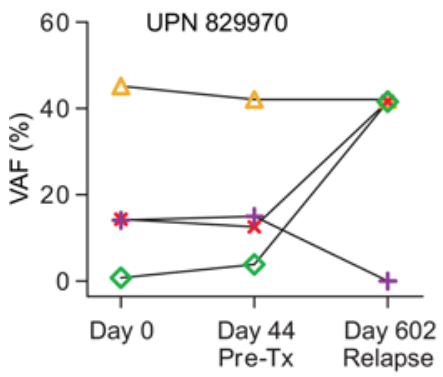

$\triangle$ Cluster 1: $A P O B$

$\times$ Cluster 2: KIAA1683

$\diamond$ Cluster 3 (no RMG)

+ Cluster 4: KCNK13
E

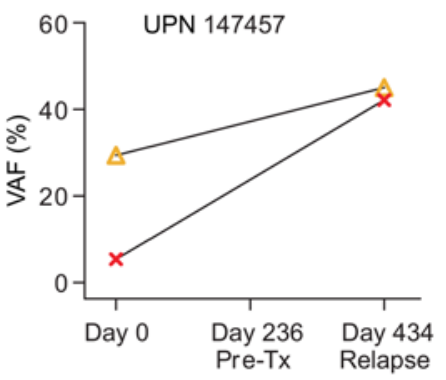

$\triangle$ Cluster 1 (19 RMGs)

$\times$ Cluster 2 (no RMG)
$\mathbf{F}$

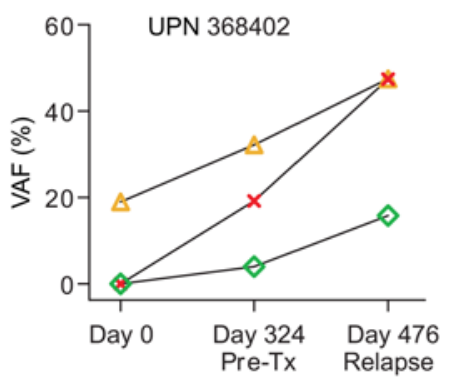

$\triangle$ Cluster 1: SRSF2, RUNX1

$\times$ Cluster 2: SF3A1

$\diamond$ Cluster 3: EPPK1
G

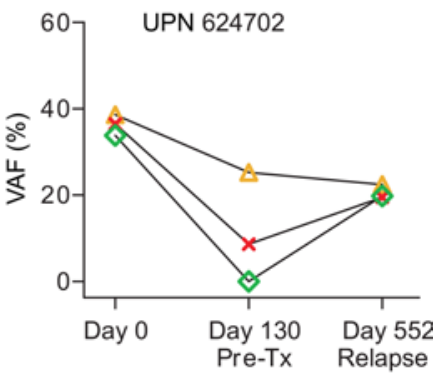

$\triangle$ Cluster 1: PMS2

$\times$ Cluster 2: SRSF2, TET2

$\checkmark$ Cluster 3: STAG2

H

Subclonal Emergence/Expansion defined by

\begin{tabular}{|l|l|l|}
\hline $\begin{array}{l}\text { SNVs } \\
\text { Indels }\end{array}$ & Both & SVs \\
& & \\
368402 & 147457 & 145094 \\
& 624702 & 280837 * \\
& 829970 & 435866 \\
& & 499258 \\
& & 574214 \\
\hline
\end{tabular}

Figure 1. Subclones expand or emerge at disease progression after allogeneic hematopoietic cell transplant. The variant allele fractions (VAFs) of all validated mutations in diploid genomic regions for UPN 829970 at relapse were compared with those at first sampling (day 0) (A) and before transplant (B). Genes known to be recurrently mutated in myeloid malignancies (RMG) are labeled. Unsupervised clustering of individual mutation VAFs (e.g., SNVs and indels) identified 4 clones, including one that expanded (green, cluster 3) and one that contracted (purple, cluster 4) at progression. Cluster 1 (founding clone) mutations were always detectable at progression. (C) Summary of clonal evolution from first sampling (day 0 ) to relapse in UPN 829970 . The founding clone contained cluster 1 mutations (yellow) that were present in approximately $90 \%$ of bone marrow cells at first sampling (day 0 ). Clone 1 gave rise to 2 subclones, as shown (cluster 2 mutations in red, $29 \%$ of cells; cluster 4 mutations in purple, $28 \%$ of cells). The subclone containing cluster 2 mutations gave rise to a rare, pretransplant subclone (cluster 3 mutations, green) that expanded and gained additional SVs to become the most abundant subclone at relapse, present in $83 \%$ of cells. Selected somatic alterations, some copy number corrected, are shown. Days are numbered relative to first sampling, and selected after transplant days are shown in parentheses. VAFs represent the summation of read counts from exome and standard validation sequencing when available. (D-G) Dynamic changes in the size of the clones in 4 subjects with expanding/emerging clones defined by SNVs/indels in diploid areas of the genome. The RMG associated with each cluster is labeled and, in some cases, copy number corrected, with the exception of data in E, due to space constraints (see Supplemental Figure 3B). (H) Summary of the type of somatic alteration associated with expanding or emerging subclones at progression after transplant. SNV, single nucleotide variant; indels, insertion or deletion; SV, structural variant; RMG, recurrently mutated gene. A CBL indel was also detected in UPN 280837 but was not able to be accurately quantified (marked with an asterisk). 
Collectively, we found that a subclone expanded or emerged at progression after alloHCT in all 9 patients (Figures 1 and 2 and Supplemental Figure 4). In the majority ( $n=7$, UPNs 145094, 280837, 368402 , 499258, 574214, 829970, 624702), the expanding/emerging subclone contained mutations in genes and/or SVs known to be associated with myeloid malignancies (Figures 1 and 2, Supplemental Figure 4, and Supplemental Tables 1, 3, and 4). In addition, we observed the following nonmutually exclusive patterns of subclonal dynamics at disease progression: (a) the expanding/emerging subclone became the most abundant clone at disease progression ( $n=7$, UPNs 147457, 280837, 368402, 624702, 829970, 499258, 574254) (Figure 1, A and C, and Supplemental Figures 3 and 4); (b) a subclone contracted with therapy, with minimal contribution to the disease progression ( $n=3$, UPNs 145094 , 280837, 829970) (Figure 1, C and D, and Supplemental Figures 3 and 4); and (c) the founding clone at first sampling was the most abundant clone at disease progression, with emergence of an additional minor subclone ( $n=2$, UPNs 145094, 435866) (Supplemental Figure 4).

Rare subclone mutations can exist before alloHCT and give rise to disease progression after alloHCT. To determine whether the emergent subclone at progression was present prior to transplant as a rare, preformed subclone, we used ultra-deep error-corrected sequencing (sensitivity of $\sim 0.1 \%$ VAF or the ability to detect a heterozygous mutation present in 1 of 500 cells). We examined cases (UPNs 368402 and 829870) in which the SNVs/indels present in a rising, progression subclone were not readily detected by standard sequencing (exome or capture based) at first sampling. In UPN 829970, none of the 4 genes in cluster 3, which define the most abundant relapse subclone, were detectable by standard sequencing at first sampling. However, with error-corrected sequencing, 3 of the 4 mutations were detected with a mean VAF of $1.83 \%$, demonstrating that the progression-associated clone was a minor clone present at first sampling that persisted throughout transplant (Figure 3A).

In contrast, UPN 368402 had 2 distinct subclones that expanded after alloHCT and were present immediately prior to transplant (mean VAF of 4\% and 19\%), but none of the 20 SNVs that define these 2 subclones were detectable by standard sequencing at first sampling, which was prior to azacitidine chemotherapy. Using ultra-deep error-corrected sequencing, these mutations remained undetectable (Figure 3B). This raises the possibility that pretransplant azacitidine therapy could have not only provided selective pressure, but also induced mutations that led to clonal evolution and progression (see below for UPN 368402).

The effect of pretransplant therapy on clonal evolution at disease progression. Six of these patients had samples that allowed us to quantify the disease burden present at the time of transplant and to characterize the effects of pretransplant therapy on the clonal evolution of MDS in patients who progress after alloHCT. Five of six patients received treatment (e.g., hypomethylating agents, cytotoxic therapy, or both) between diagnosis and transplant (UPNs 145094, 280837, 368402, 435866, 624702) (Supplemental Table 1 and Supplemental Figure 4). First, we observed marked discordance between myeloblast counts and estimates of tumor proportion based on sequencing in these pretransplant samples. Five of the six patients had a myeloblast count that underestimated the proportion of tumor cells in their bone marrow. The median percentage of tumor cells in these 6 patients before transplant was $57.5 \%$ (range, 5\%-85.5\%) based on sequencing results, while the median blast count was 3.5\% (range, 1\%-9\%) (Figure 4A).

Next, we observed that subclones that responded to pretransplant therapy can eventually expand to drive clonal evolution at disease progression. For example, in UPN 624702, what appeared to be the founding clone at first sampling was actually an admixture of 3 clones, each with differential sensitivities to pretransplant chemotherapy (Figure 4B). The subclone that reexpanded at progression was undetectable by standard sequencing at the pretransplant time point and underwent further clonal evolution at progression after alloHCT by acquiring a SV [t(12;18)(p13;q21), Figure 4B and Supplemental Table 1]. Tracking serial samples was necessary to resolve the clonal complexity present in this patient.

Finally, we observed that pretransplant therapy can induce mutations that may directly impact disease progression. For example, subclonal mutation clusters 2 and 3 emerged before transplant after 8 cycles of azacitidine therapy in UPN 368402. These mutations were not detectable at first sampling (Figure $3 \mathrm{~B}$ ) and were enriched for C-to-G transversions, including coding C-to-G transversions in the recurrently mutated genes $S F 3 A 1$ and EPPK1 in distinct subclones (Figure 4, C and D). Consistent with this finding, we have previously observed a similar increase in acquired C-to-G transversions in emerging subclones from patients following decitabine treatment (Supplemental Figure 7) $(1,10,26)$. C-to-G transversions are known to be induced by the hypomethylating agent decitabine $(10,27)$, directly implicating the pretransplant therapy in shaping the mutation spectrum prior to transplant. 
A

UPN 574214
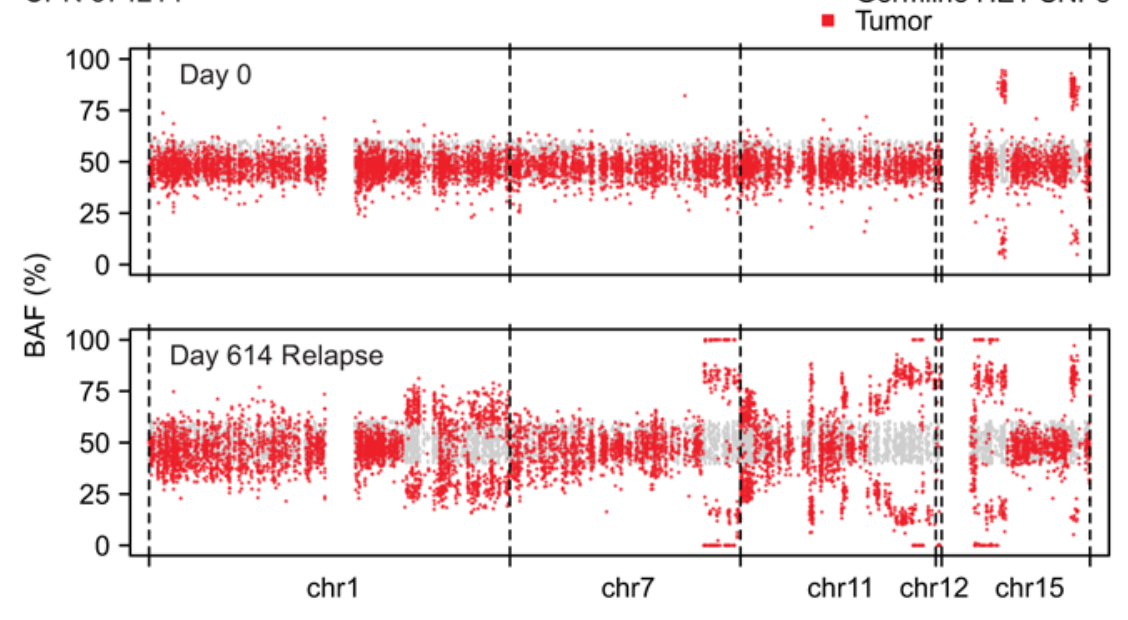

Chromosomes

C

UPN 574214

Day $\Rightarrow$

0
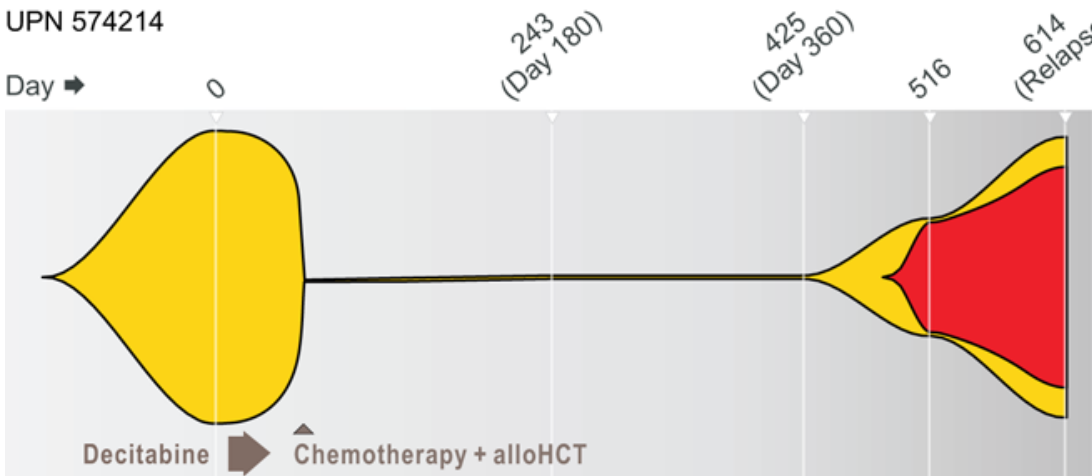

BOD1L, TP53, del 5q, del 17p

$\operatorname{del} 7 q$, other SVs ${ }^{\star}$

D

UPN 829970

- Germline HET SNPs

- Tumor
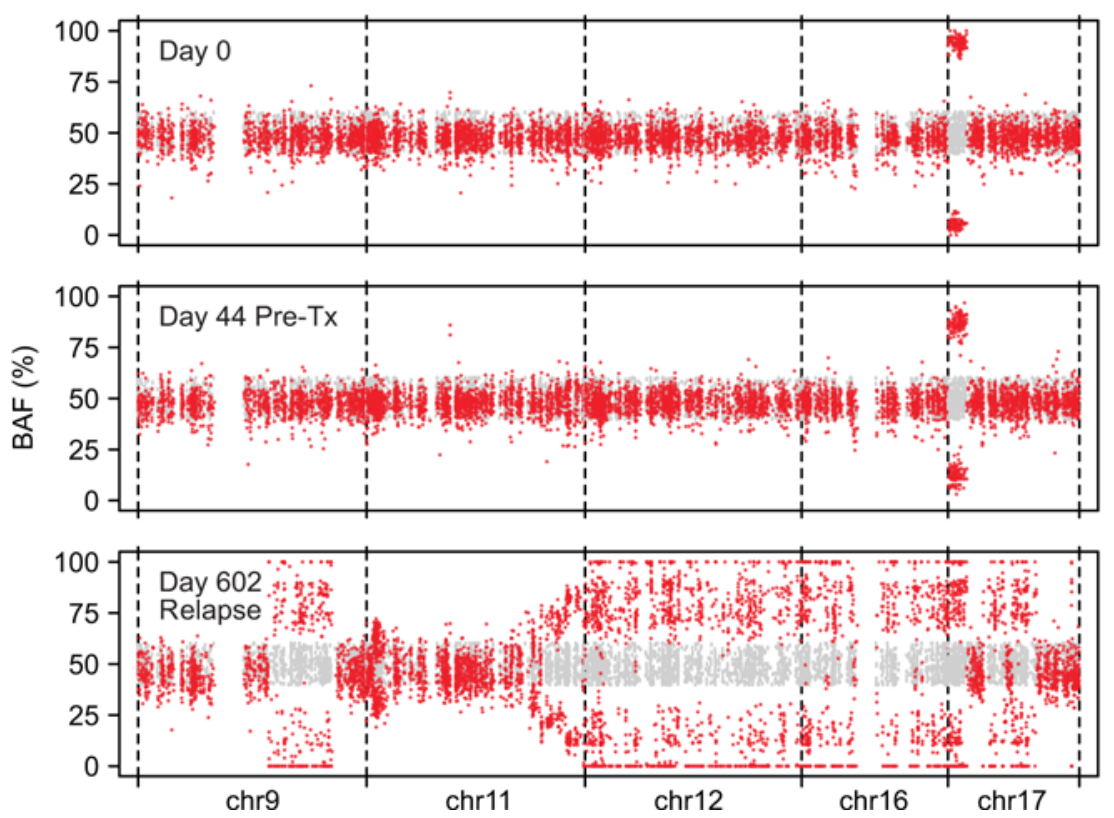

B
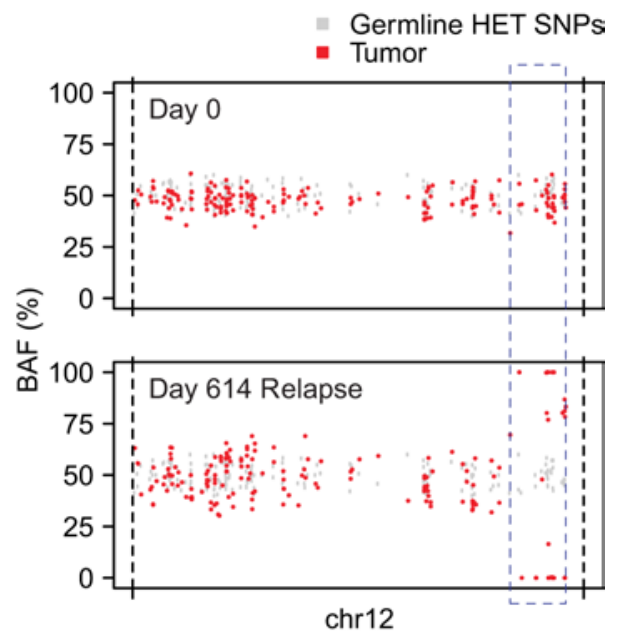
Figure 2. Structural variants contribute to clonal evolution after allogeneic hematopoietic cell transplant. Dynamic changes in the B allele frequency (BAF) (reference allele determined by NCBI refseq) in MDS cells for selected chromosomes at first sampling (day 0) and after transplant for UPN 574214 (A and B) and UPN 829970 (D). The BAF was determined using heterozygous (HET) SNPs identified in normal tissue (skin) by exome sequencing (gray). The BAF was then plotted for MDS samples (red). Divergence of the BAF away from $50 \%$ is directly proportional to the percentage of cells harboring loss of heterozygosity (LOH) due to a copy number alteration or uniparental isodisomy (Supplemental Table 4). Posttransplant samples were sorted for MDS myeloblasts to reduce donor SNP contamination. (C) Clonal evolution of UPN 574214. Cells harboring the founding clone mutations (cluster 1, yellow) escaped eradication and gave rise to a subclone gaining new structural variants, not detectable prior to transplant, which expanded into the most abundant subclone after transplant, present in approximately $60 \%$ of cells (red). The percentage of cells harboring the structural variants (SV) was estimated by using the formula ( 50 - mean deleted allele frequency) $\times 2$. The mean variant allele frequency (VAF) of single nucleotide variants (SNVs) at disease progression was $39.5 \%$ (present in $~ 79 \%$ cells), thus the subclone harboring these SV changes was the most abundant clone at progression. Selected somatic genetic alterations are shown, including a subset that were copy number corrected. LOH on chromosomes 1, 11, 12, and 15 (marked with an asterisk). Pre, pretransplant.

Serial tracking of tumor burden after alloHCT using sequencing. We next asked whether somatic mutations could be detected prior to overt clinical progression or relapse, including at the early time points of day 30 and day 100 after transplant. Using capture probes targeting all SNVs and indels identified by EES at first sampling and progression (with standard sequencing), we interrogated 28 serial banked samples at various time points after alloHCT (plus the known relapse samples) (Supplemental Table 2 and Supplemental Figure 8) and correlated the sequencing results with clinical evidence of disease. We also used error-corrected sequencing to confirm the presence of SNVs detected by capture-based standard sequencing to determine whether rare mutations (i.e., present in as few as 1 in 500 cells) could be detected prior to clinical disease progression.

In the 8 patients who had bone marrow samples harvested at day 30 and 100 after alloHCT, 6 of them had detectable mutations present at one or both time points (UPNs 145094, 280837, 368402, 435866, 147457, 499258) (Figure 5A). In 2 cases (UPN 829970 and 624702), MDS-associated variants were not detected using error-corrected sequencing in the bone marrow obtained during routine clinical follow-up. In 4 instances, (in 2 patients, UPN 145094 and 574214) standard sequencing and/or error-corrected sequencing detected low-level mutation burdens prior to clinical evidence of disease (Figure 5B and Supplemental Tables 1 and 5). In 3 of these 4 instances, mutations were only detectable by error-corrected sequencing. For example, in UPN 145094, there was no evidence of morphologic disease at day 30 (day 196 after first sampling), and no mutations were detected by standard sequencing. However, error-corrected sequencing detected 10 of 20 interrogated mutations (mean VAF $=0.13 \%$ ). By day 100 , although there was still no evidence of disease clinically, mutations were detected by both standard and error-corrected sequencing (VAFs of $1.07 \%$ and $0.86 \%$, respectively).

Persistence of mutations detected using sequencing may also serve as an adjunct to standard clinical testing in cases of clinical equipoise, such as determining whether low-level mixed chimerism represents normal recipient or diseased recipient marrow. Error-corrected sequencing confirmed the presence of MDS-associated SNVs in 6 patients when the bone marrow showed $<5 \%$ blasts and low-level mixed chimerism ( $10 \%$ or less) (Supplemental Tables 1 and 5$)$. There were no cases where there was evidence of mixed donor chimerism or return of cytogenetic/FISH abnormalities in which error-corrected sequencing failed to detect MDS-associated SNVs (including 1 case in which standard sequencing failed to detect MDS-associated SNVs) (Supplemental Table 5). In the samples where SNVs were detectable months to years prior to overt clinical progression, the detectable mutations always included founding clone mutations (e.g., cluster 1), although the number of detectable mutations varied, perhaps due to sensitivity of sequencing as the disease burden approached the lower limits of detection.

\section{Discussion}

In this study, we used comprehensive genomic analysis to describe the subclonal evolution and clonal selection that contributes to disease relapse after alloHCT in MDS. We made several observations that have clinical implications. First, in all 9 cases, emergence or expansion of a subclone was evident at disease progression, implicating subclone expansion as a potential biomarker of subsequent progression. Second, SVs were present in the emergent/expanding subclone in the majority ( 8 of 9) of patients, implicating the acquisition of new SVs as late subclonal events that could contribute to progression. Third, mutations (SNVs and/or indels) detected in the founding clone at first sampling were always present at disease progression after alloHCT, making them ideal candidates to track tumor burden serially. Finally, mutations were often detectable at low levels at days 30 and 100 after alloHCT prior to clinical progression. 
A

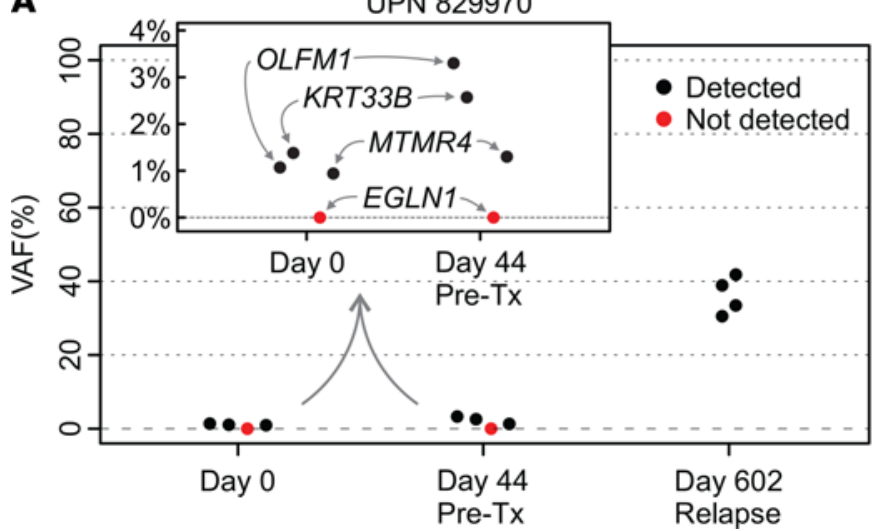

B

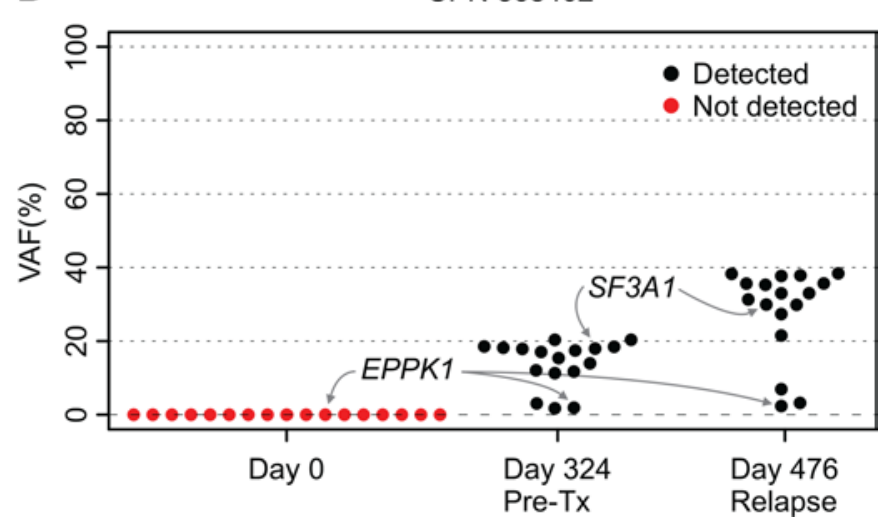

Figure 3. Rare subclones and therapy-emergent subclones can be detected using ultra-deep error-corrected sequencing. Mutations that were detectable at progression, but not first sampling, by standard sequencing were subjected to ultra-deep error-corrected sequencing. Mutation variant allele fractions (VAFs) detected by error-corrected sequencing are shown. (A) In UPN 829970, 3 of the 4 mutations that define the most abundant relapse clone were detected (shown in black) at first sampling (day 0 ) using error-corrected sequencing, but not standard sequencing, consistent with the most abundant clone at progression after transplant being partially formed at diagnosis. These variants define cluster 3 (green) in Figure 1, A-D. (B) In UPN 368402, 20 mutations present in 2 distinct subclones that were identified by standard sequencing at after transplant progression, but not at first sampling, were interrogated by error-corrected sequencing at first sampling (day 0 ), immediately pretransplant (Pre-Tx, after azacitidine therapy), and after transplant (Relapse). None of the mutations were detected at first sampling, consistent with mutation acquisition occurring during azacitidine therapy. SF3A1 is harbored in cluster 2 (red) in Figure 1F and Figure 4D. EPPK1 is harbored in cluster 3 (green) in Figure 1F and Figure 4D. The limit of detection for error-corrected sequencing is approximately $0.1 \%$ VAF. This approach is about 10 times more sensitive than deep Illumina sequencing ( 1\%-2\% VAF sensitivity). At day 0 , 3 of the 20 mutations were not successfully interrogated, including SF3A1 (Supplemental Methods and Supplemental Table 3).

While the sample size was small, the majority of cases (7 of 9) acquired genetic changes in the expanding/emerging subclones (e.g., SNVs, indels, and/or SVs) in genes or loci known to be associated with myeloid malignancies. These results likely underestimate the complexity of acquired events that contribute to disease pathogenesis, since changes in the transcriptome, methylome, and proteome may play roles in clonal outgrowth at progression and were not investigated here. Although bulk populations of myeloblasts were sequenced, rather than single cells, we do not expect that this affected our interpretation of clonal dynamics, as it has been shown that the subclonal architecture imputed from sequencing bulk material is equivalent to single cell genotyping $(28,29)$. We did not observe deletions or UPD involving the immunoregulatory genes (e.g., $H L A, P D-L 1$, or $P D-L 2$ genes), as previously observed following relapse after haploidentical alloHCT (30). However, we cannot rule out the possibility that other mechanisms of immune escape by tumor cells contributed to clonal evolution and disease progression after alloHCT. Future studies could address whether immune escape contributes to or cooperates with genetic drivers of progression.

We identified tumor heterogeneity (i.e., the presence of at least 1 subclone) in the majority of patients prior to alloHCT. We observed that minor subclones present at diagnosis, or a minor subclone that is not cleared by before alloHCT therapy, can undergo further genetic evolution and drive progression after alloHCT. We also show that the alterations in the mutation spectrum in subclones that expand after alloHCT are associated with pretransplant therapy. For example, pretransplant therapy with azacitidine was characterized by the acquisition of C-to-G transversions, which occurred in genes known to be associated with AML/MDS. This is not restricted to azacitidine, as we observed a similar enrichment in the acquisition of C-to-G transversions following decitabine treatment, suggesting that hypomethylating agents may influence the clonal evolution of subclones.

We consistently observed the acquisition of new SVs as late genetic events in expanding/emerging subclones. Although our cohort includes several patients with therapy-related MDS, our findings are consistent with other reports that have shown that cytogenetic lesions are acquired as late events in MDS (4). In our series, the SVs characterizing the expanding/emergent subclone at progression could only be unequivocally detected prior to transplant in $25 \%$ of cases. These SVs may have occurred in rare cells before alloHCT that were below the level of detection, or they may have occurred as a result of conditioning regimens or increased genomic instability. Future studies involving an expanded number of de novo MDS cases that are capable of tracking SVs using sensitive sequencing-based assays may help resolve these possibilities.

We included genetic analysis of the bone marrow just prior to transplant in 6 cases to examine the impact of MDS-associated clonal burden at the time of transplant. Although there are no prospective studies examin- 
A

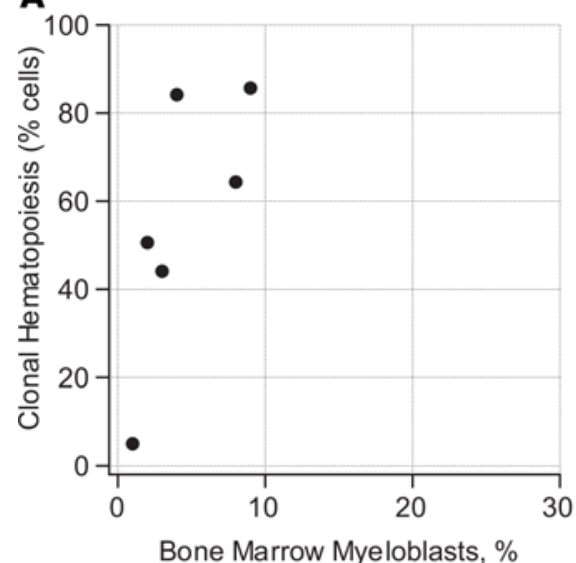

C

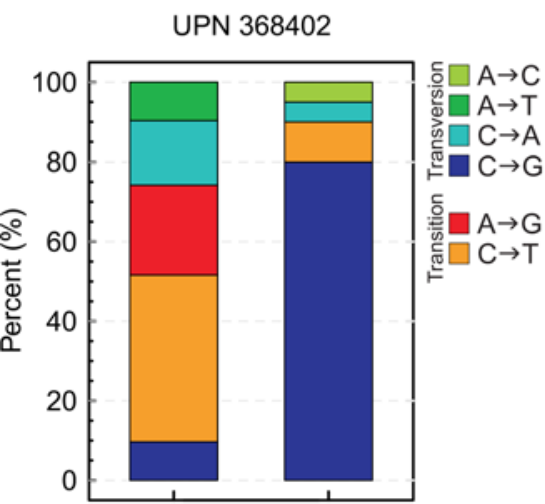

Cluster 1 Cluster 2, 3

\section{B}

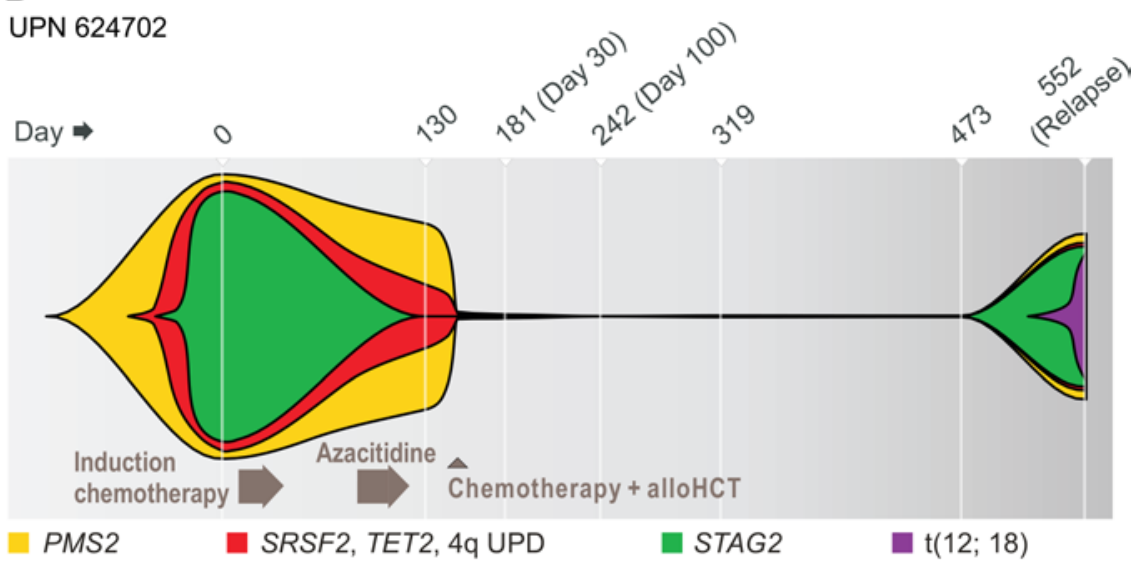

D

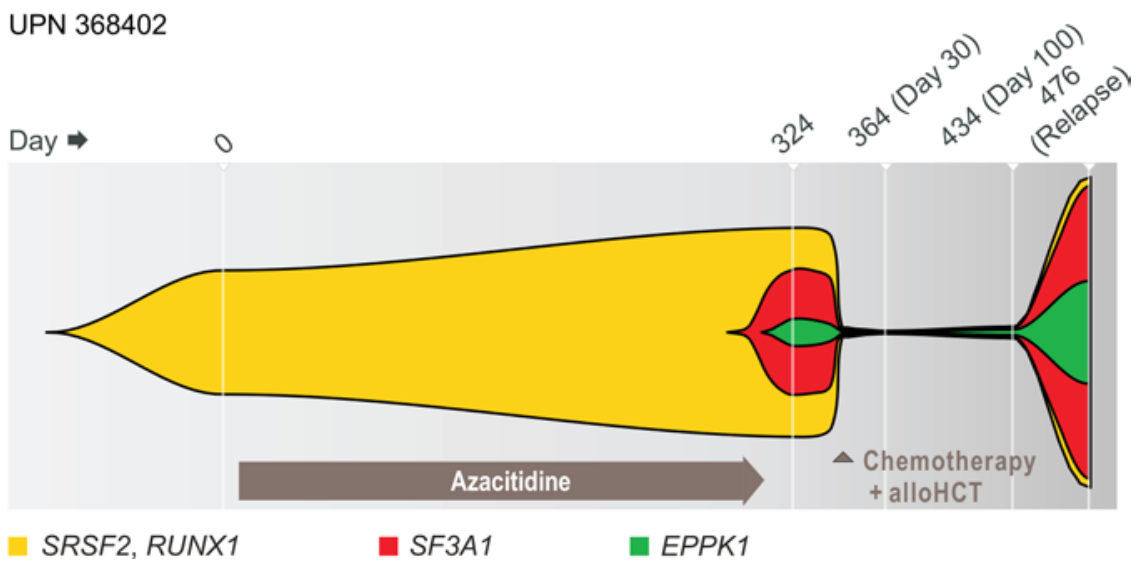

Figure 4. Preallogeneic hematopoietic cell transplant therapy affects clonal evolution at disease progression. (A) The bone marrow myeloblast percentage underestimates MDS tumor burden, as determined by the percentage of cells harboring MDS variants detected by standard sequencing, at the time of transplant. (B) Summary of the clonal evolution of UPN 624702. Linear clonal evolution occurs when cells in the founding clone (containing cluster 1 mutations, yellow) give rise to clone 2 with the addition of cluster 2 mutations (red) and, subsequently, clone 3 with the addition of cluster 3 mutations (green). All mutations present in clones 1-3 are present at first sampling (day 0). After treatment with induction therapy and azacitidine, clone 3 contracts and is no longer detectable by standard sequencing by day 130, but clones 1 and 2 remain. A very rare cell from clone 3 (harboring cluster 1,2 , and 3 mutations) escapes eradication by transplant and gains a structural variant to emerge as the most abundant clone at progression (purple). Selected somatic alterations, some copy number corrected, are shown. Days are numbered as in Figure 1. (C) Spectrum of single nucleotide variant base substitutions present in cluster 1 at first sampling (yellow in D) and cluster 2 and 3 (red and green in D, respectively) present after azacitidine treatment in UPN 368402 . Clusters 2 and 3 show a greater proportion of C-to- $C$ transversions, consistent with azacitidine-induced mutations. (D) Summary of the clonal evolution of UPN 368402 . Only the founding clone, shown in yellow, is detected at first sampling. After 8 cycles of azacitidine therapy, 2 new subclones emerge, with clone 2 (shown in red) derived from the founding clone, and clone 3 (green) derived from clone 2 . Clone 2 expands to become the most abundant clone at progression after transplant.

ing the impact of debulking MDS prior to transplant, several large retrospective studies have suggested that the percentage of bone marrow blasts at the time of transplant influences outcome (15-17); expert panels currently recommend cytoreductive therapy prior to alloHCT when $\geq 10 \%$ myeloblasts exist (14). Measuring tumor burden in pretransplant samples using founding clone VAFs may provide a more predictive endpoint in clinical trials compared with the blast count. Studies of minimal identifiable disease (MID) determined by multiparameter flow cytometry show that the effect of pretransplant MID status on outcome depended on intensity of conditioning regimen (31). In our series, relapse occurred in patients who received both myeloablative and reducedintensity conditioning regimens. Future studies involving larger number of patients are needed to adequately address the predictive ability of pretransplant MDS clonal burden, as measured by VAFs, on outcomes in the setting of myeloablative and reduced-intensity conditioning regimens.

We show that low-level mutation burden can often be detected at day 30 and day 100 after alloHCT, important early clinical milestones. Detection of only a subset of the founding clone mutations at tumor reemergence may be due to technical limitations of our assays. Further studies will be needed to deter- 
A

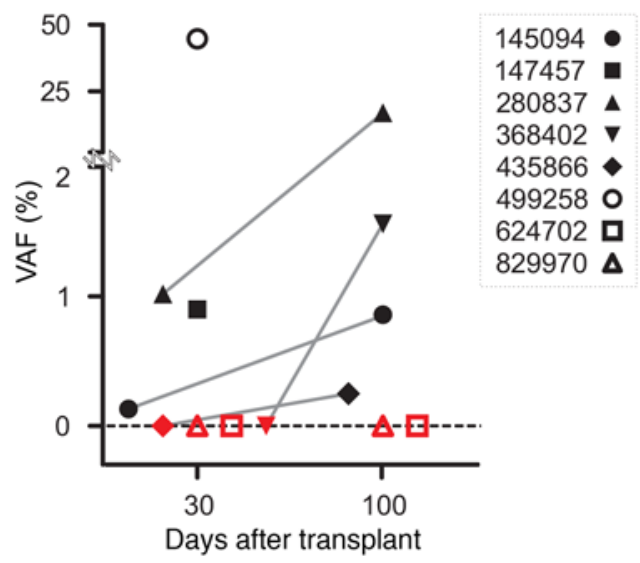

B

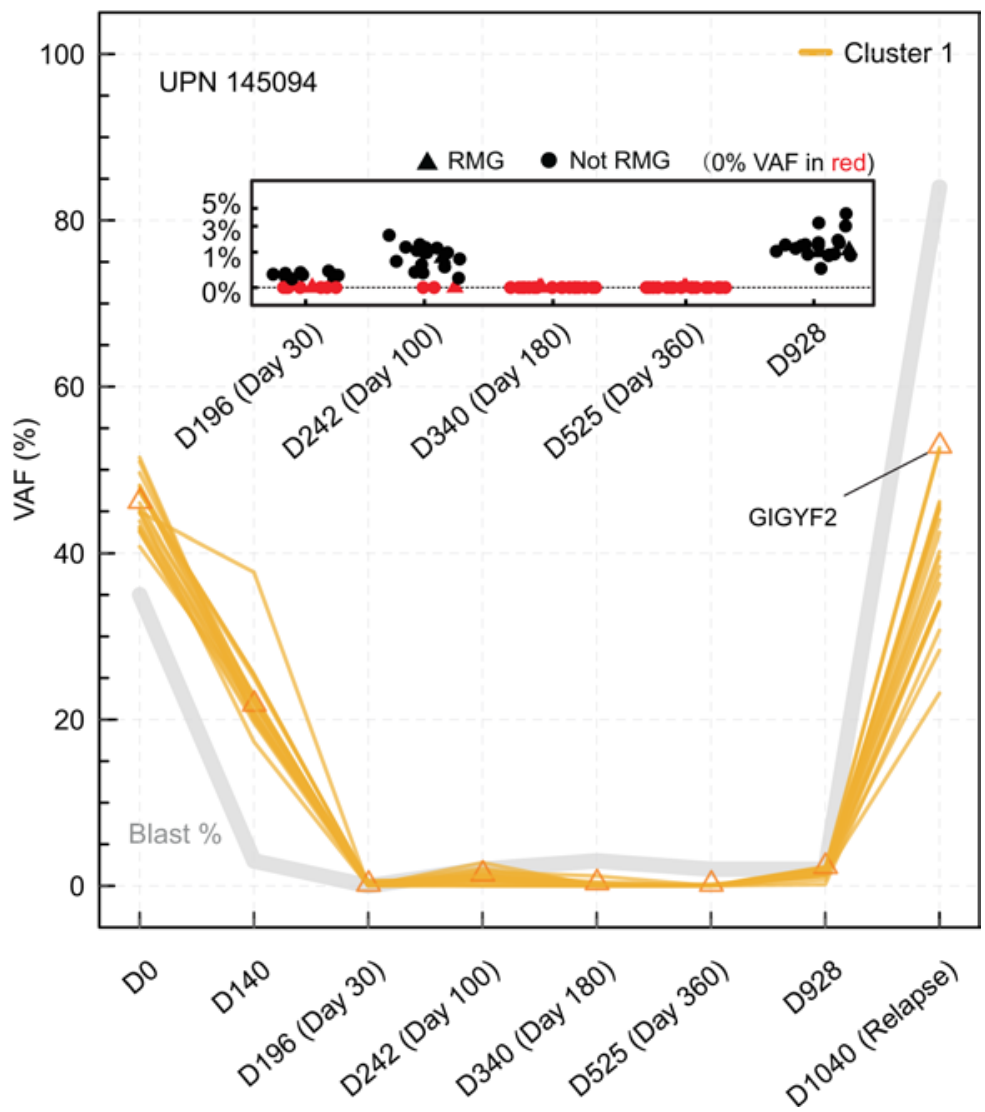

Figure 5. Serial tracking of tumor burden during therapy and after allogeneic hematopoietic cell transplant. (A) Somatic mutations (SNVs/indels) identified by standard sequencing were interrogated in samples day 30 and day 100 after transplant by ultra-deep error-corrected sequencing. Six of eight patients had detectable variants at day 30 and/or day 100. Samples with no detectable variants are shown in red at day 30 or day 100. (B) Detection of mutation VAFs in UPN 145094 at serial time points during therapy with standard sequencing. VAFs represent the summation of read counts from exome and standard validation sequencing when available. Days are numbered relative to the days after first sampling (day 0), and selected days after transplant are shown in parentheses. The inset shows serial tracking of somatic mutations at various times after transplant for UPN 145094 samples by ultra-deep error-corrected sequencing. Variants that are detectable are shown in black, and undetectable variants are shown in red. Mutations were detected at days 196 and 242 (days 30 and 100 after transplant, respectively) and day 928 by error-corrected sequencing. RMG, recurrently mutated genes (i.e., 285 genes known to be recurrently mutated in myeloid malignancies).

mine whether detection of MDS-associated variants at day 30 and 100 after alloHCT is predictive for relapse and will need to include sequencing of samples from patients that do not relapse after alloHCT. Defining the threshold of clonal cells that are predictive of future progression and determining whether prospectively monitoring after transplant patients is relevant for predicting early MDS reemergence or disease progression will be important going forward.

In conclusion, MDS progression after alloHCT is characterized by subclonal expansion and mutational evolution, which can be influenced by pretransplant therapy with hypomethylating agents. Although progression is characterized by increasing genetic diversity (including acquisition of SVs), the founding clone mutations were uniformly present at progression, suggesting that tracking founding clone mutations may be useful to detect impending progression/relapse.

\section{Methods}

Patients and samples. We enrolled 9 patients with a history of MDS or therapy-related MDS who had disease progression following an alloHCT at Washington University in St. Louis, St. Louis, Missouri, USA. All patients had available DNA from bone marrow and skin (as a source of normal DNA) before alloHCT and bone marrow at progression. Patients received either myeloablative $(n=3)$ or reduced-intensity $(n=6)$ conditioning (Supplemental Table 5). Disease progression was defined as $>5 \%$ myeloblasts in the marrow, loss of donor chimerism prompting an intervention by the treating physician, or reemergence of pretransplant cyto- 
genetic abnormalities. Tumor DNA was sourced from myeloblasts isolated from the relapse samples using flow cytometric cell sorting to minimize contamination by donor cells (except for UPN 147457, where only unsorted DNA was available). Myeloblasts were isolated from the progression sample after alloHCT using flow cytometry to isolate cells in the blast gate (CD45 $5^{\mathrm{dim}}$, side scatter low) and CD $34^{+}$if available.

Sequencing and SNP arrays. DNA from paired normal skin and bone marrow was enriched for all the coding exons from a custom set of 285 recurrently mutated MDS and AML genes (RMG, ref. 10) and "spikedin" to an exome capture sequencing reagent (enhanced exome sequencing [EES]), as previously described (10). Exome sequencing data was deposited in the dbGAP database (phs000159.v9). Enriched libraries were sequenced on a HiSeq2000 instrument 2 times at a read length of $101 \mathrm{bp}$, as previously described (10). In order to validate all putative somatic variants identified by EES, we designed an array of custom-capture probes (Roche NimbleGen) to cover all putative variants present in any sample from the 9 patients. Hybridization, sequence production, and variant calling for validation are described in the Supplemental Methods and are previously described (6). All samples were sequenced deeply, with mean tumor coverage of 246 times in the exomes, 1,146 times over key MDS genes, and 537 times in the validation sequencing (Supplemental Figure 1). In addition, we performed ultra-deep error-corrected sequencing on skin samples, before alloHCT, and after alloHCT serial treatment bone marrow, as previously described (10). Briefly, ligation-based amplification probes were designed to target both DNA strands of all SNV mutations identified by EES. DNA (500 ng) was digested and hybridized to probes plus 10-bp degenerate oligonucleotides (unique molecular identifiers) and sample-specific indexes. Cases were sequenced to target 20,000 times to 100,000 times total coverage on a HiSeq 4000. Data were analyzed using the Barcrawler analysis pipeline (Eric J. Duncavage, unpublished data), with a maximum sensitivity of approximately $0.1 \%$ (additional details are provided in the Supplemental Methods). CNA analysis using sequencing and SNP arrays is described in the Supplemental Methods. To assess the contribution of CNAs/LOH to clonal evolution, we identified heterozygous SNPs in the normal skin and determined whether $\mathrm{LOH}$ was evident in the bone marrow sample due to a somatic amplification, deletion, or UPD. To differentiate among these possibilities, we intersected LOH data and copy number analysis. Divergence of a heterozygous SNP frequency (i.e., B-allele frequency) away from 50\% is directly proportional to the percentage of cells harboring the $\mathrm{LOH}$ (Supplemental Methods). A complete list of the various genomic assays performed on patient samples and time points is provided in Supplemental Table 2.

Statistics. We calculated whether each detected variant was above the background "noise" level on a per-assay and position-by-position basis as follows: For each variant, read counts were gathered from all other samples (tumor and normal) at the same location. Samples with VAF above $20 \%$ were excluded from the background calculation. A $P$ value was obtained via Fisher's exact test, comparing the reference and variant reads at a site to the number of reference and variant reads in all other samples. Multiple testing correction was applied with the $\mathrm{R}$ p.adjust function (default parameters) and then variants with an adjusted $P$ value of less than 0.1 were retained. The process was then repeated iteratively, with subsequent background calculations excluding all variants retained in previous rounds. This was repeated until no new variants were identified ( 3 rounds). $P$ values of less than 0.05 were considered significant.

Study approval. Specimens were obtained after subjects provided written informed consent and enrolled on a protocol approved by the institutional review board at Washington University in St. Louis.

\section{Author contributions}

DCL, JFD, PW, TJL, TAG, and MJW designed the research studies. MAJ, EJD, JR, RSF, CCF, and MO conducted experiments. MAJ, EJD, GSC, CAM, JS, KE, JR, RSF, SEH, IP, and JSW acquired and analyzed data. MAJ, EJD, GSC, CAM, TAG, and MJW wrote the manuscript.

\section{Acknowledgments}

This work was supported by a SPORE in Leukemia (P50CA171963 to DCL and EJD), the Institute of Clinical and Translational Sciences-Clinical and Translational Science Awards (to EJD), Gabrielle's Angel Foundation (to MJW), a Leukemia and Lymphoma Society Scholar Award and Quest for CURES Award (to MJW), an American Society of Hematology Scholar Award (to MAJ), and the Lottie Caroline Hardy Trust (to TAG and MJW). Support for procurement of human samples was provided by an NIH/National Cancer Institute grant (P01 CA101937). Technical assistance was provided by the Alvin J. Siteman Cancer Center Tissue Procurement Core, supported by an National Cancer Institute Cancer Center grant (P30CA091842). We thank Richard K. Wilson for helpful scientific discussions. 
Address correspondence to: Matthew J. Walter, Washington University School of Medicine, 660 S. Euclid Avenue, Campus Box 8007, St. Louis, Missouri, 63110, USA. Phone: 314.362.9409; Email: mjwalter@wustl.edu.

1. Walter MJ, et al. Clonal architecture of secondary acute myeloid leukemia. N Engl J Med. 2012;366(12):1090-1098.

2. Ding L, et al. Clonal evolution in relapsed acute myeloid leukaemia revealed by whole-genome sequencing. Nature. 2012;481(7382):506-510.

3. Wong TN, et al. Role of TP53 mutations in the origin and evolution of therapy-related acute myeloid leukaemia. Nature. 2015;518(7540):552-555.

4. Mossner M, et al. Mutational hierarchies in myelodysplastic syndromes dynamically adapt and evolve upon therapy response and failure. Blood. 2016;128(9):1246-1259.

5. Jacoby MA, Duncavage EJ, Walter MJ. Implications of tumor clonal heterogeneity in the era of next-generation sequencing. Trends Cancer. 2015;1(4):231-241.

6. Walter MJ, et al. Clonal diversity of recurrently mutated genes in myelodysplastic syndromes. Leukemia. 2013;27(6):1275-1282.

7. Papaemmanuil E, et al. Clinical and biological implications of driver mutations in myelodysplastic syndromes. Blood. 2013;122(22):3616-3627.

8. Haferlach T, et al. Landscape of genetic lesions in 944 patients with myelodysplastic syndromes. Leukemia. 2014;28(2):241-247.

9. Makishima H, et al. Dynamics of clonal evolution in myelodysplastic syndromes. Nat Genet. 2017;49(2):204-212.

10. Uy GL, et al. Dynamic changes in the clonal structure of MDS and AML in response to epigenetic therapy. Leukemia. 2017;31(4):872-881

11. Deeg HJ, et al. Five-group cytogenetic risk classification, monosomal karyotype, and outcome after hematopoietic cell transplantation for MDS or acute leukemia evolving from MDS. Blood. 2012;120(7):1398-1408.

12. Brierley CK, Steensma DP. Allogeneic stem cell transplantation in myelodysplastic syndromes: does pretransplant clonal burden matter? Curr Opin Hematol. 2016;23(2):167-174.

13. Mielcarek M, Storer BE, Flowers ME, Storb R, Sandmaier BM, Martin PJ. Outcomes among patients with recurrent high-risk hematologic malignancies after allogeneic hematopoietic cell transplantation. Biol Blood Marrow Transplant. 2007;13(10):1160-1168.

14. de Witte T, et al. Allogeneic hematopoietic stem cell transplantation for MDS and CMML: recommendations from an international expert panel. Blood. 2017;129(13):1753-1762.

15. Della Porta MG, et al. Predictive factors for the outcome of allogeneic transplantation in patients with MDS stratified according to the revised IPSS-R. Blood. 2014;123(15):2333-2342.

16. Warlick ED, Cioc A, Defor T, Dolan M, Weisdorf D. Allogeneic stem cell transplantation for adults with myelodysplastic syndromes: importance of pretransplant disease burden. Biol Blood Marrow Transplant. 2009;15(1):30-38.

17. Sierra J, et al. Bone marrow transplantation from HLA-identical siblings as treatment for myelodysplasia. Blood. 2002;100(6):1997-2004.

18. Bejar R, et al. Somatic mutations predict poor outcome in patients with myelodysplastic syndrome after hematopoietic stem-cell transplantation. J Clin Oncol. 2014;32(25):2691-2698.

19. Lindsley RC, et al. Prognostic mutations in myelodysplastic syndrome after stem-cell transplantation. N Engl J Med. 2017;376(6):536-547.

20. Yoshizato T, et al. Genetic abnormalities in myelodysplasia and secondary acute myeloid leukemia: impact on outcome of stem cell transplantation. Blood. 2017;129(17):2347-2358.

21. Della Porta MG, et al. Clinical effects of driver somatic mutations on the outcomes of patients with myelodysplastic syndromes treated with allogeneic hematopoietic stem-cell transplantation. J Clin Oncol. 2016;34(30):3627-3637.

22. Woo J, et al. Mutational analysis in serial marrow samples during azacitidine treatment in patients with post-transplant relapse of acute myeloid leukemia or myelodysplastic syndromes. Haematologica. 2017;102(6):e216-e218.

23. Gomez-Ferreria MA, et al. Human Cep192 is required for mitotic centrosome and spindle assembly. Curr Biol. 2007;17(22):1960-1966.

24. Vardiman JW, et al. The 2008 revision of the World Health Organization (WHO) classification of myeloid neoplasms and acute leukemia: rationale and important changes. Blood. 2009;114(5):937-951.

25. Arber DA, et al. The 2016 revision to the World Health Organization classification of myeloid neoplasms and acute leukemia. Blood. 2016;127(20):2391-2405.

26. Welch JS, et al. TP53 and decitabine in acute myeloid leukemia and myelodysplastic syndromes. $N$ Engl J Med. 2016;375(21):2023-2036.

27. Jackson-Grusby L, Laird PW, Magge SN, Moeller BJ, Jaenisch R. Mutagenicity of 5-aza-2'-deoxycytidine is mediated by the mammalian DNA methyltransferase. Proc Natl Acad Sci USA. 1997;94(9):4681-4685.

28. Klco JM, et al. Functional heterogeneity of genetically defined subclones in acute myeloid leukemia. Cancer Cell. 2014;25(3):379-392.

29. Hughes AE, et al. Clonal architecture of secondary acute myeloid leukemia defined by single-cell sequencing. PLoS Genet. 2014;10(7):e1004462.

30. Vago L, et al. Loss of mismatched HLA in leukemia after stem-cell transplantation. N Engl J Med. 2009;361(5):478-488.

31. Festuccia M, et al. Minimal identifiable disease and the role of conditioning intensity in hematopoietic cell transplantation for myelodysplastic syndrome and acute myelogenous leukemia evolving from myelodysplastic syndrome. Biol Blood Marrow Transplant. 2016;22(7):1227-1233. 